Research Article

\title{
The Stability Conditions for a Heavy Solid Motion
}

\author{
A. I. Ismail $\mathbb{D}^{1,2}$ \\ ${ }^{1}$ Mechanics Department, Faculty of Engineering and Islamic Architecture, Umm Al-Qura University, P. O. Box 5555, \\ Makkah, Saudi Arabia \\ ${ }^{2}$ Department of Mathematics, Faculty of Science, Tanta University, Tanta, P. O. Box 31527, Egypt
}

Correspondence should be addressed to A. I. Ismail; aiismail@uqu.edu.sa

Received 14 September 2020; Revised 25 September 2020; Accepted 10 October 2020; Published 24 October 2020

Academic Editor: E. M. Khalil

Copyright (C) 2020 A. I. Ismail. This is an open access article distributed under the Creative Commons Attribution License, which permits unrestricted use, distribution, and reproduction in any medium, provided the original work is properly cited.

\begin{abstract}
In this paper, the stability conditions for the rotary motion of a heavy solid about its fixed point are considered. The center of mass of the body is assumed to lie on the moving $z$-axis which is assumed to be the minor axis of the ellipsoid of inertia. The nonlinear equations of motion and their three first integrals are obtained when the principal moments of inertia are distributed as $I_{1}<I_{2}<I_{3}$. We construct a Lyapunov function $L$ to investigate the stability conditions for this motion. We give a numerical example to illustrate the necessary and sufficient conditions for the stability of the body at certain moments of inertia. This problem has many important applications in different sciences.
\end{abstract}

\section{Introduction}

The problem of stability of a rotary motion of a heavy solid moving in a uniform gravity field or a rigid body moving in a Newtonian one is considered as one of the vital problems in the nonlinear dynamics. These problems were searched in the last half century. These problems are classified according to the bodies containing partially fluid or fully fluid or lie in attracting centers. In [1], the rotational motion about the center of mass for an asymmetric dynamic body with an aspheric cavity filled with liquid is studied. Numerical analysis for changing the kinetic momentum vector of the body was given. The extreme position for the stability of the axis of rotation of a body was obtained. A mass geometry solid of the Earth was attained. In [2], the authors studied the stability conditions of rotary motion for a heavy solid containing cavity filled fully or partially with a viscous fluid. The author in [3] considered the stability problem for a steady rotary motion of a gyro filled with liquid in a cavity. The author achieved the conditions of stability for this case of studied motion. The authors in [4] studied a branching for the stability of permanent rotatory motions of a rigid body filled with a viscous fluid. In [5], the stability problem of a permanent rotary motion of an asymmetric top about a fixed point under the influence of a central Newtonian force field is considered for different cases. In [6], the evolution of the stability for the rotary motion of a planet containing a liquid core is considered. For some special cases of the orbiting motions for a planet, the stability conditions are obtained in [7]. The work of [8] gave the conditions for stability of a rotating unperturbed rigid body about a fixed point in different problems using the well-known energy first integral.

Moreover, the first integral related to energy was used in [8] for the aim of obtaining sufficient conditions of stability when the governing system of the body has not any perturbation. In [9], the authors evaluated the orbiting for a satellite containing a cavity filled with a viscous fluid. For both the uniform and the Newtonian force fields, the problems of stabilities of the orbiting bodies about fixed points are considered in some steady motion cases in [10]. In [11], the stability spin for a Lagrange top with linear oscillators is considered. In [12], the authors evaluated the plane periodic rotary motion stabilities of a symmetric rigid body moving about a fixed point when the principal moments of inertia $I_{1}=I_{2}=2 I_{3}$. The permanent orbiting stability problem of an asymmetric gyro is considered in [13] when the gyro moved under a Newtonian force field. In [14], the author considered the conditions of stability motion of a 
rigid body rotating about the $x$-axis when $I_{1}>I_{2}>I_{3}$. He assumed a moment $\lambda_{1}$ about the $x$-axis and deduced the equations of motion. There are some new applications of nano-/microcomponents related to stability conditions in different application subjects that should be mentioned here. The applications such as the Chebyshev-Ritz technique for static stability and vibration analysis of nonlocal microstructure-dependent nanostructures are given by Ebrahimi et al. [15].

Our article is searching the conditions of stability of a nonperturbed coupled heavy solid rotating about a fixed point in a uniform field of gravity. The body is assumed to rotate about the minor $z$-axis of the ellipsoid of inertia with couple $\lambda_{3}$. We consider the body rotates with principal moments of inertia to satisfy the condition $I_{1}<I_{2}<I_{3}$. We use the three well-known first integral of the problem for finding the stability conditions using a modified definition of Lyapunov function $L$. A numerical example is considered to show the stability conditions for a certain case.

\section{Definition of the Problem}

In this section, we define the problem of the motion of a heavy solid rotating about the minor $z$-axis of the ellipsoid of inertia. Consider the coordinate frame $O X Y Z$ is the fixed coordinate system in space and $O x y z$ is the fixed system in the body. Let the body rotates with an angular velocity vector $\underline{\omega}=\left(\omega_{1}, \omega_{2}, \omega_{3}\right)$ to the system $O x y z$. Assume the couple $\underline{\lambda}=$ $\left(0,0, \lambda_{3}\right)$ to the moving coordinate system Oxyz. Let the mass center of the body lies on the $z$-axis (see Figure 1), and the moments of inertia of the body satisfy the conditions:

$$
I_{1}<I_{2}<I_{3}, \quad z_{0} \neq 0,
$$

where $\left(0,0, z_{0}\right)$ is the position of the mass center for the origin.

Thus, the nonlinear system of equations of motion for this case and its first integrals are obtained as follows:

$$
\begin{gathered}
I_{1} \dot{\omega}_{1}+\left(I_{3}-I_{2}\right) \omega_{2} \omega_{3}+\lambda_{3} \omega_{2}=-m g z_{0} \gamma_{2}, \\
I_{2} \dot{\omega}_{2}+\left(I_{1}-I_{3}\right) \omega_{3} \omega_{1}-\lambda_{3} \omega_{1}=m g z_{0} \gamma_{1}, \\
I_{3} \dot{\omega}_{3}+\left(I_{2}-I_{1}\right) \omega_{1} \omega_{2}=0 \\
\dot{\gamma}_{1}=\omega_{3} \gamma_{2}-\omega_{2} \gamma_{3}, \\
\dot{\gamma}_{2}=\omega_{1} \gamma_{3}-\omega_{3} \gamma_{1}, \\
\dot{\gamma}_{3}=\omega_{2} \gamma_{1}-\omega_{1} \gamma_{2}, \\
I_{1} \omega_{1}^{2}+I_{2} \omega_{2}^{2}+I_{3} \omega_{3}^{2}-2 m g z_{o} \gamma_{3}=E_{1}, \\
I_{1} \omega_{1} \gamma_{1}+I_{2} \omega_{2} \gamma_{2}+\left(I_{3} \omega_{3}+\lambda_{3}\right) \gamma_{3}=H_{1}, \\
\gamma_{1}^{2}+\gamma_{2}^{2}+\gamma_{3}^{2}=1,
\end{gathered}
$$

where the unit vector in the direction of the $Z$-axis is $\underline{\widehat{Z}}=\left(\gamma_{1}, \gamma_{2}, \gamma_{3}\right), m$ is the solid mass, $g$ is the gravity acceleration, $\cdot \equiv \mathrm{d} / \mathrm{d} t$ is the differentiation to time, and $E_{1}$ and $H_{1}$ are the initial energy and momentum constants. Assuming that $m g z_{0}=a$, equation (2) can be rewritten as follows:

$$
\begin{array}{r}
I_{1} \dot{\omega}_{1}=\left(I_{2}-I_{3}\right) \omega_{2} \omega_{3}-\lambda_{3} \omega_{2}-a \gamma_{2}, \\
I_{2} \dot{\omega}_{2}=\left(I_{3}-I_{1}\right) \omega_{3} \omega_{1}+\lambda_{3} \omega_{1}+a \gamma_{1}, \\
I_{3} \dot{\omega}_{3}=\left(I_{1}-I_{2}\right) \omega_{1} \omega_{2} .
\end{array}
$$

Assuming that the following special solutions satisfy the six nonlinear differential equations of motion (5) and (3),

$$
\begin{array}{r}
\omega_{1}=\omega_{2}=\gamma_{1}=\gamma_{2}=\lambda_{3}=0, \\
\omega_{3}=r=\text { const }, \\
\gamma_{3}=1 .
\end{array}
$$

This is a case of uniform rotation of the solid about the minor axis of the ellipsoid of inertia ( $z$-axis).

\section{Stability Investigation}

In this section, the stability procedure of the unperturbed solid is investigated. Consider that the variables in (6) change according to

$$
\begin{aligned}
\omega_{1} & =\Gamma_{1}, \\
\omega_{2} & =\Gamma_{2}, \\
\omega_{3}= & +\Gamma_{3}, \\
\gamma_{1} & =\xi_{1}, \\
\gamma_{2} & =\xi_{2}, \\
\gamma_{3}=1 & +\xi_{3} .
\end{aligned}
$$

Substituting the variables in (7) into the equations of motion (5) and (3), it gives

$$
\begin{array}{r}
I_{1} \dot{\Gamma}_{1}=\left(I_{2}-I_{3}\right) \Gamma_{2}\left(r+\Gamma_{3}\right)-a \xi_{2}, \\
I_{2} \dot{\Gamma}_{2}=\left(I_{3}-I_{1}\right)\left(r+\Gamma_{3}\right) \Gamma_{1}+a \xi_{1}, \\
I_{3} \dot{\Gamma}_{3}=\left(I_{1}-I_{2}\right) \Gamma_{1} \Gamma_{2}, \\
\dot{\xi}_{1}=\left(r+\Gamma_{3}\right) \xi_{2}-\Gamma_{2}\left(1+\xi_{3}\right), \\
\dot{\xi}_{2}=\Gamma_{1}\left(1+\xi_{3}\right)-\left(r+\Gamma_{3}\right) \xi_{1}, \\
\dot{\xi}_{3}=\Gamma_{2} \xi_{1}-\Gamma_{1} \xi_{2} .
\end{array}
$$

Substituting the variables in (7) into the first integrals (4), it gives

$$
\begin{array}{r}
V_{1}=I_{1} \Gamma_{1}^{2}+I_{2} \Gamma_{2}^{2}+I_{3} \Gamma_{3}^{2}+2 I_{3} r \Gamma_{3}-2 a \xi_{3}, \\
V_{2}=I_{1} \Gamma_{1} \xi_{1}+I_{2} \Gamma_{2} \xi_{2}+I_{3} \Gamma_{3} \xi_{3}+I_{3} r \xi_{3}, \\
V_{3}=\xi_{1}^{2}+\xi_{2}^{2}+\xi_{3}^{2}+2 \xi_{3},
\end{array}
$$

where $V_{1}, V_{2}$, and $V_{3}$ are constants.

We make a linear combination of the relations in (9) to satisfy the Lyapunov and Chetayev stability procedures. the Lyapunov function $L$ is modified to be in the form:

$$
L=V_{1}-2 r V_{2}+\left(I_{3} r^{2}+a\right) V_{3} .
$$

Thus, using the relations in (9), we obtain

$$
L=L_{1}+L_{2}+L_{3} \text {, }
$$




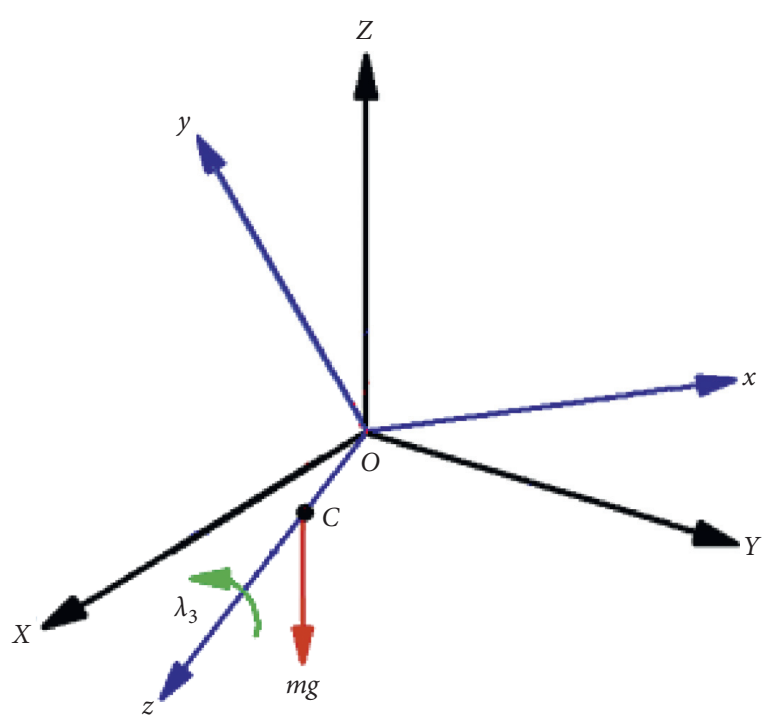

Figure 1: Description of forces.

where

$$
\begin{aligned}
& L_{1}=I_{1} \Gamma_{1}^{2}-2 I_{1} r \Gamma_{1} \xi_{1}+\left(I_{3} r^{2}+a\right) \xi_{1}^{2}, \\
& L_{2}=I_{2} \Gamma_{2}^{2}-2 I_{2} r \Gamma_{2} \xi_{2}+\left(I_{3} r^{2}+a\right) \xi_{2}^{2}, \\
& L_{3}=I_{3} \Gamma_{3}^{2}-2 I_{3} r \Gamma_{3} \xi_{3}+\left(I_{3} r^{2}+a\right) \xi_{3}^{2} .
\end{aligned}
$$

We deduce from the relations in (12) that $L_{i}>0, i=1,2,3$, if and only if the following inequalities are satisfied [14], respectively:

$$
\begin{aligned}
& \left|\begin{array}{cc}
I_{1} & -I_{1} r \\
-I_{1} r & \left(I_{3} r^{2}+a\right)
\end{array}\right|=I_{1}\left(I_{3} r^{2}+a\right)-I_{1}{ }^{2} r^{2}>0, \\
& \left|\begin{array}{cc}
I_{2} & -I_{2} r \\
-I_{2} r & \left(I_{3} r^{2}+a\right)
\end{array}\right|=I_{2}\left(I_{3} r^{2}+a\right)-I_{2}{ }^{2} r^{2}>0, \\
& \left|\begin{array}{cc}
I_{3} & -I_{3} r \\
-I_{3} r & \left(I_{3} r^{2}+a\right)
\end{array}\right|=I_{3}\left(I_{3} r^{2}+a\right)-I_{3}{ }^{2} r^{2}=I_{3} a>0 .
\end{aligned}
$$

Thus, we obtain from the back substitution

$$
\begin{aligned}
a & >0, \\
I_{3} & >I_{2}, \\
I_{3} & >I_{1} .
\end{aligned}
$$

We note that when condition (1) is satisfied, inequalities (13) and (14) are investigated automatically.

Thus, the conditions in (13) considered the sufficient and necessary conditions for the unperturbed stability motion of the solid concerning the variables $\omega_{1}, \omega_{2}, \omega_{3}, \gamma_{1}, \gamma_{2}$, and $\gamma_{3}$.

\section{A Simple Application Example}

A simple application example can be given in this section. Just formulation the sufficient and necessary conditions for the unperturbed stability motion are studied.
Consider a rigid body of mass $M$ with moments of inertia as follows:

$$
\begin{aligned}
I_{1} & =10 \mathrm{~kg} \cdot \mathrm{m}^{2}, \\
I_{2} & =15 \mathrm{~kg} \cdot \mathrm{m}^{2}, \\
I_{3} & =20 \mathrm{~kg} \cdot \mathrm{m}^{2}, \\
M & =100 \mathrm{~kg}, \\
g & =9.81 \mathrm{~m} \cdot \mathrm{s}^{-2}, \\
z_{o} & =50 \mathrm{~m}, \\
r & =10 \mathrm{rad} \cdot \mathrm{s}^{-1} .
\end{aligned}
$$

From (15), we deduce that

$$
\begin{gathered}
a=49050 \mathrm{kgm}^{2} \cdot \mathrm{s}^{-2}, \\
\left(I_{3} r^{2}+a\right)=51050 .
\end{gathered}
$$

From (13), (14), and (15), we deduce that

$$
\begin{aligned}
I_{1}\left(I_{3} r^{2}+a\right)-I_{1}{ }^{2} r^{2} & =500500>0, \\
I_{2}\left(I_{3} r^{2}+a\right)-I_{2}{ }^{2} r^{2} & =743250>0, \\
I_{3} a & =981000>0 .
\end{aligned}
$$

From this example, we deduce that if condition (1) is satisfied by (15), $L_{i}>0, i=1,2,3$, is satisfied if and only if relation (17) is satisfied.

\section{Conclusion}

The necessary and sufficient conditions for the stability of the rotary motion of a solid about a fixed point are considered when the center of mass of the body lies on the minor $z$-axis of the ellipsoid of inertia. The system of equations of motion and their first integrals are obtained. The modified Lyapunov function $L$ is constructed depending on the three first integrals. The necessary and sufficient conditions are constructed in the case $I_{3}>I_{2}>I_{1}$ that the $x$-axis is the major axis of the ellipsoid of inertia and the $y$-axis is the intermediate axis while the $z$-axis is the minor one. The moments of inertia $I_{\mathrm{i}}, i=1,2,3$ are assumed as arbitrary constants that satisfy the above condition, and $\lambda_{3}$ is estimated by substituting (7) into the second equation from (4). This problem has wide applications in satellite motions which assumed moments of inertia according to the above relations [16-18]. A numerical example is given to show the stability conditions for a special case.

\section{Data Availability}

Data sharing not applicable to this article as no datasets were generated or analyzed during the current study.

\section{Conflicts of Interest}

The author declares he has no conflicts of interest. 


\section{References}

[1] A. L. Rachinskaya, "Motion of a solid body with cavity filled with viscous liquid," Cosmic Research, vol. 53, no. 6, pp. 476-480, 2015.

[2] V. Z. Osipov and R. S. Sulikashvili, "Oscillation of a solid body with a spherical cavity filled fully with a viscous fluid on an elliptic orbit," Trudy Tbilis, vol. 58, pp. 175-186, 1978.

[3] F. L. Chernous'ko, Motion of a Solid Body with Cavities Filled with a Viscous Fluid, VTs ANSSSR, Moscow, Russia, 1968.

[4] F. L. Chernous'ko, "Motion of a solid with cavities filled with a viscous fluid at small Reynolds numbers," Zh Vychisl Mat Fiz, vol. 5, no. 6, pp. 1049-1070, 1965.

[5] E. P. Smirnova, "Stabilization of the free rotation of an asymmetric top with a cavity filled fully with a viscous fluid," Prikl Mat Mekh, vol. 33, no. 6, pp. 980-985, 1974.

[6] V. V. Sidorenko, "Evolution of rotational motion of a planet with a liquid core," Astron Vestn, vol. 27, no. 2, pp. 119-127, 1993.

[7] V. G. Vil'ke and A. V. Shatina, "Evolution of a rotating satellite with a cavity filled with a viscous fluid," Kosm Issled, vol. 31, no. 6, pp. 22-30, 1993.

[8] N. S. Sidorenkov, Physics of the Earth's Rotation Instabilities, Nauka/Fizmatlit, Moscow, Russia, 2002.

[9] L. D. Akulenko, D. D. Leshchenko, and A. L. Rachinskaya, "Evolution of rotations of a satellite with a cavity filled with a viscous fluid," Mekh. Tverd. Tela, vol. 37, pp. 126-139, 2007.

[10] Y. V. Abrarova, "The stability of the steady motions of a rigid body in a central field," Journal of Applied Mathematics and Mechanics, vol. 59, no. 6, pp. 903-910, 1995.

[11] Y. N. Kononov, "Spin stability of a Lagrange top containing linear oscillators," Journal of Mathematical Sciences, vol. 103, no. 1, pp. 38-42, 2001.

[12] B. S. Bardin and A. A. Savin, "The stability of the plane periodic motions of a symmetrical rigid body with a fixed point," Journal of Applied Mathematics and Mechanics, vol. 77, no. 6, pp. 578-587, 2013.

[13] M. Iñarrea, V. Lanchares, A. I. Pascual, and A. Elipe, "Stability of the permanent rotations of an asymmetric gyrostat in a uniform Newtonian field," Applied Mathematics and Computation, vol. 293, pp. 404-415, 2017.

[14] W. S. Amer, "The necessary and sufficient condition for the stability of a rigid body," Journal of Advances in Physics, vol. 13, no. 6, pp. 4999-5003, 2017.

[15] F. Ebrahimi, M. R. Barati, and O. Civalek, "Application of Chebyshev-Ritz method for static stability and vibration analysis of nonlocal microstructure-dependent nanostructures," Engineering with Computers, vol. 36, no. 3, pp. 953-964, 2020.

[16] L. D. Akulenko, D. D. Leshchenko, and A. L. Rachinskaya, "Evolution of the satellite fast rotation due to the gravitational torque in a dragging medium," Mechanics of Solids, vol. 43, no. 2, pp. 173-184, 2008.

[17] L. D. Akulenko, Y. S. Zinkevich, D. D. Leshchenko, and A. L. Rachinskaya, "Rapid rotations of a satellite with a cavity filled with viscous fluid under the action of moments of gravity and light pressure forces," Cosmic Research, vol. 49, no. 5, pp. 440-451, 2011.

[18] A. L. Rachinskaya and A. N. Dutsa, "Evolution of fast rotations of a satellite due to perturbation torques," Visnik Odes'k. NATS, vol. 17, pp. 175-184, 2012. 